\title{
How image and text semantic analysis systems can be applied for educational and teaching purposes
}

\section{Jozef Stašák $k^{*}$}

\begin{abstract}
This contribution deals with algorithm closely related to design and implementation of simple database conceptual models based on terms and principles concerned to image and text semantic analysis, while the term deterministic image is being postulated and introduced and its structure elements are described. On the other hand, the presented contribution contains a set of adequate information, how that algorithm may be applied in teaching concerned to simple database conceptual model.
\end{abstract}

Keywords: image semantic analysis, deterministic databases, non-deterministic databases.

\section{Introduction}

We live in the age of information presented to the user in different forms and via various approaches, methods and techniques. The information presented in the form of applied text, static or dynamic image, sonic or multimedia documents. On the other hand, such types of information may be combined and applied within a machine readable record or document. As a result of that, two principal questions may be postulated: How such document content can be understood and interpreted in relation to the document user's or reader's requirements? What approaches, methods and techniques should be applied for these purposes?

When looking for the answer to the above-mentioned questions we can consult materials closely related to computational linguistics (Bolshakov - Gelbukh), visual literacy (The Visual Literacy White Paper), the content analysis of text (Eakins - Graham) or image from semantic point of view (Harris) where algorithms based on the fuzzy set apparatus play a role of great importance (Košir

Jozef Stašák, Dubnica Technological Institute, Dubnica nad Váhom, Slovakia; jozefstasak@yahoo.com 
- Tašic; Niemeyer - Canty). However, there is a set of images with the structure which may be described via the set of deterministic relations, and which are denoted as deterministic images, while the types of images with the structure and behavior which shall be described by stochastic or heuristic formulas or relations are considered to be non-deterministic images (see also Section 2.2).

The aim of this paper is to establish or create the basis for quantification terms and principles closely related to structure, features and functionality of deterministic images with respect to design and implementation of those algorithms and application programs subsequently, which help the students to make proper simple database conceptual model. In order to achieve such an objective, several partial and subordinated aims should be fulfilled. They are postulated as follows:

- Quantification of deterministic images related to their internal and external structure elements.

- Proposal of principles related to design and implementation of simple database with the application of formulas and relations postulated within the abovementioned quantification.

This paper consists of two principal sections. The first section (Section 2) consists of three subsections $(2.1,2.2$, and 2.3). The Section 2.1 deals with terms and principles related to semantic text and image analysis, and the image (static image) is considered to be a stochastic or heuristic system. The Section 2.2 deals with quantification of deterministic images related to their internal and external structure elements, and the Section 2.3 deals with problems related to how the Image and Text Semantic Analysis Systems should be applied for learning and teaching purposes. Subsequently, these problems are further developed in Section 3.

1 How the image and text semantic analysis systems may be applied for educational and teaching purposes?

\subsection{Terms and principles related to the standard image and text semantic analysis systems}

Terms and principles related to the text semantic analysis

Any record or document, the content of which is represented by Text in Natural Language (hereinafter as TNL Document) consists of semantic subsets creating its principle structure elements. Such semantic subsets are called fragments and contain natural language sentences. Any idea represented in natural language is considered to 


\section{Acta Technologica Dubnicae}

volume 1, 2011, issue 1

be a logical sentence. Furthermore, each logical sentence consists of objects and semantic relations, while those relations may be categorized as follows:

- Semantic relations who provide interactions among objects, objectives, living species, products, events and processes within the appropriate objects (Type A Relation).

- Semantic relations which provide interactions among objects and within TNL sentence (Type B Relation).

- Semantic relations who provide interactions among lists and reference databases (Type C Relation).

It means these relations are considered to be multifunctional. Objects and Relations of Type A and B are related to TNL internal structure and fragments and Type C Relation are concerned to TNL external structure (Stašák, 2004). Fragments' objects and semantic relations are concerned the TNL content structure, however TNL content is represented by its own lifecycle which is represented by phases postulated as follows:

- TNL-decomposition, while a set of adequate fragments is created and the fragments are categorized with respect to appropriate objectives and species;

- Extraction of words and phrases which are considered to be objects and relations. These objects are divided into three principal groups:

- Terms to be explained (hereinafter as Tbe terms);

- Principal terms (hereinafter as Pet terms), while they represent existing terms which enable generating Tbe terms;

- Relating terms (hereinafter as Ret terms), while they represent relations among objects represented by Pet terms especially.

- Creation of lists (fragments and documents) and their categorization according to objects.

- Representation of structure related to lists and creation of semantic networks (Stašák, 2004).

The above-mentioned TNL structure and life-cycle elements create the basis for further development of TNL semantic analysis with the use of algorithms based on fuzzy set apparatus described within subsequent articles and contributions (Stašák, 2006).

\section{Terms and principles related to the image semantic analysis}

There are many approaches, methods and techniques related to the Image Semantic Analysis. However, only two approaches will be discussed in this section. The first approach originates in traditional methods based on standard image indexing; however problems with method type application have led to the rise of interest in techniques for 


\section{Acta Technologica Dubnicae}

volume 1, 2011, issue 1

retrieving images on the basis of automatically-derived features such as color, texture and shape - a technology now generally referred to as Content-Based Image Retrieval (CBIR). Current indexing practice for images relies largely on text descriptors or classification codes, supported in some cases by text retrieval packages designed or adapted specially to handle images. Again, remarkably little evidence on the effectiveness of such systems has been published. User satisfaction with such systems appears to vary considerably.

CBIR operates on a totally different principle from keyword indexing. Primitive features characterizing image content, such as color, texture and shape are computed for both stored and query images, and used to identify (say) the 20 stored images most closely matching the query. Semantic features such as the type of object present in the image are harder to extract, though this remains an active research topic. Video retrieval is a topic of increasing importance - here, CBIR techniques are also used to break up long videos into individual shots, extract still key frames summarizing the content of each shot, and search for video clips containing specified types of movement (Eakins - Graham).

The second approach is based on image content segmentation and their representation with the use of algorithms based on fuzzy set apparatus. There exist many different formal approaches which postulate the actually investigated image into segments, while the central objects within the play an important role (Košir - Tašic; Niemeyer Canty). An approach related to creation of meaningful image objects is based on existence of image structure. Image segments represent structure units on the first hierarchic level; however each image segment consists of objects having principal semantic meaning. These objects are called clusters and segments having a supplementary semantic meaning are called gasps.

Segments, clusters and gasps are considered to be the principal semantic units, when considering an image semantic structure. On the other hand, sets of verbal text strings - terms, which describe the principal semantic content of clusters or gasps form semantic point of view. The terms which describe the principal semantic content of clusters or gasps are called terms to be explained (Tbe-terms, principal terms (Petterms) and relating terms (Ret-terms) - see also previous section. However, the semantic content of clusters and gasps is modeled via fuzzy sets as well (Stašák, 2004). Segments, clusters and gasps are considered to be the principal semantic units, when considering an image semantic structure. On the other hand, sets of verbal text strings - terms describe the principal semantic content of clusters or gasps form semantic point of view. Those principles have been developed within the work closely related to 
image databases (Stašák, 2004a; 2004b; 2005) and will create the basis for further sections of the paper.

\subsection{Algorithm which enables generation of deterministic image structure elements}

Internal and external structure of deterministic images and non-deterministic images

In general, any image structure may be represented by segments; cluster and gasp (see also Section 2.1). On the other hand, the cluster and gasp structure may have deterministic, stochastic or heuristic behavior. The images, clusters and gasps which have deterministic behavior are denoted as deterministic images, while the images, clusters and gasps of stochastic or heuristic behavior are denoted as non-deterministic images.

A set of database tables and relations which create an entire database may be considered to be deterministic image. However, one of the database tables shall be considered to be the leading database table which is closely related to other (subordinated) database tables and has a segment status, while the subordinated database tables are considered to be clusters from the image semantic analysis point of view. This concept of database structure is denoted as the database external structure (see also Figure1).

Any image segment consists of clusters and gasps, while an such image cluster may be represented by unique database table, the content created by a primary key, set of foreign keys and attributes defined by the user or designer. This concept of database table structure is denoted as database table internal structure (see also Figure 2). 


\section{Acta Technologica Dubnicae}

volume 1, 2011, issue 1

Figure 1 Database external structure

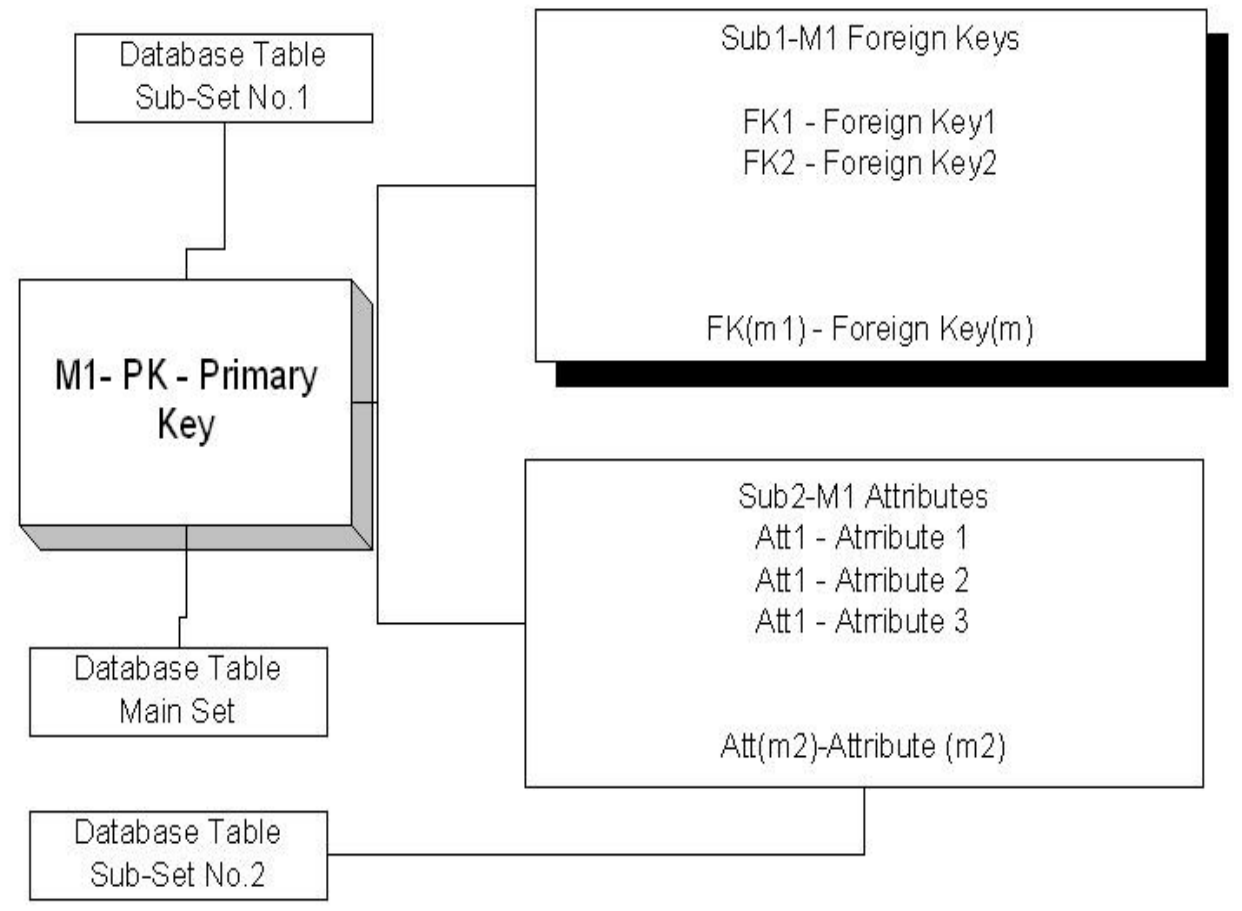


Figure 2 Database table internal structures

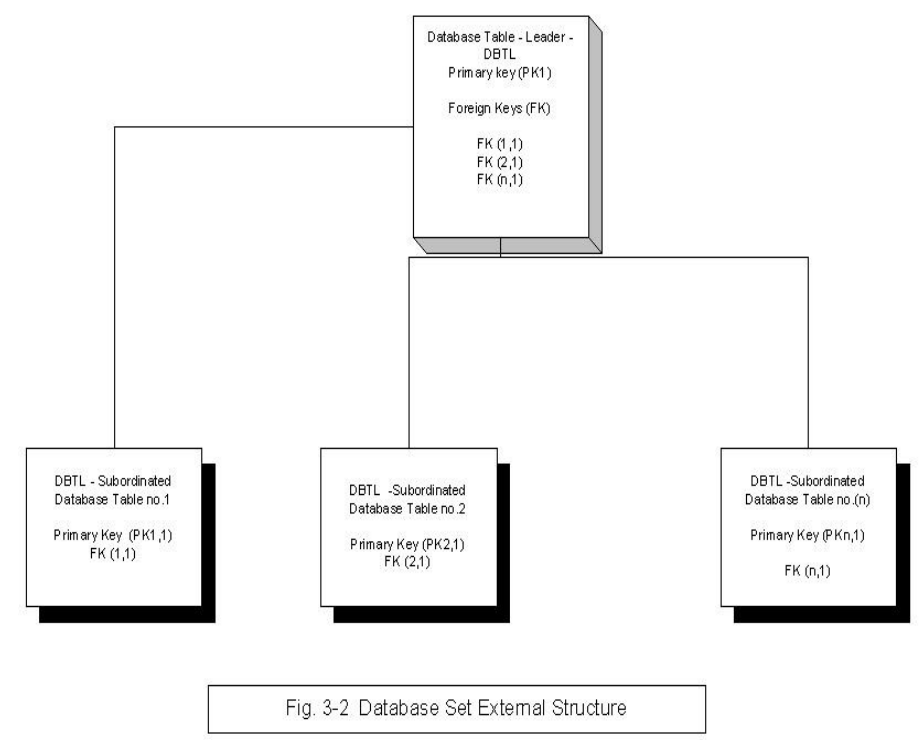

Quantification of deterministic images related to their internal and external structure elements

However, the clusters represent only one set of structure elements related to any image. The second important part of the image structure is created by elements denoted as gasps. When considering deterministic images closely related to database structure representation, the gasps may be represented by appropriate relations. Let us try doing a quantitative representation of database structure (database conceptual model) with the use of rules closely related to image content semantic analysis. Such representation is based on several considerations.

\section{Consideration No. 1}

Let us consider an image which consists of segment sets interconnected by set of appropriate relations, while the segment sets have an adequate pre-defined structure, e.g. database tables. Any database table content is created by the primary key, a number of foreign keys and attributes which represent database content. On the other hand, the primary key and the foreign keys play a role of principal importance, when creating adequate relations among the pre-defined database tables. 


\section{Acta Technologica Dubnicae}

volume 1, 2011, issue 1

In other words, the segments represented by database tables have a pre-defined structure represented by a set of attributes including the primary key and foreign keys. On the other hand, they are mutually interconnected, while the set of appropriate principles and rules shall be respected there. With respect, the above-mentioned considerations on such a segment may be represented by the set which consists of three sub-sets (see also Figure 1), while the first subset contains elements denoted as keys (primary key) the second subset contains (an appropriate number of foreign keys) and the second one contains an appropriate number of different type attributes which represent the database semantic content alone. Both of the above-mentioned subsets create a total database table semantic content.

However, a database table is considered to be an image with strictly pre-defined internal structure and the image is considered to be an integral part of any larger image which represents the entire database. The image is considered to be the sub-image of the database image (see also Figure 2).

\section{Consideration No. 2}

Let us have applied an approach related to semantic structure of image based on existing Image Segments and Clusters (Stašák, 2004).

$\operatorname{Img}=\{\operatorname{Sg}(1), \operatorname{Sg}(2) \ldots \ldots \ldots . . . \operatorname{Sg}(n)\}$

$\mathrm{m}_{1}$ - number of clusters within one image segment

$\mathrm{m}_{2}-$ number of gasps within one image segment

$\operatorname{Sg}(1)=\left\{[(\mathrm{Cl}(1,1), \operatorname{Gsp}(1,1))],[(\mathrm{Cl}(1,2), \operatorname{Gsp}(1,2))] \ldots \ldots \ldots \ldots \ldots . . .\left[\left(\mathrm{Cl}\left(1, \mathrm{~m}_{1}\right)\right.\right.\right.$, $\left.\left.\mathrm{Gsp}\left(1, \mathrm{~m}_{2}\right)\right)\right]$

$\mathrm{Sg}(2)=\left\{[(\mathrm{Cl}(2,1), \mathrm{Gsp}(2,1))],[(\mathrm{Cl}(2,2), \mathrm{Gsp}(2,2))] \ldots \ldots \ldots \ldots \ldots . . .\left[\left(\mathrm{Cl}\left(2, \mathrm{~m}_{1}\right)\right.\right.\right.$, $\left.\left.\operatorname{Gsp}\left(2, \mathrm{~m}_{2}\right)\right)\right]$

$\operatorname{Sg}(\mathrm{n})=\{[(\mathrm{Cl}(\mathrm{n}, 1), \operatorname{Gsp}(\mathrm{n}, 1))],[(\mathrm{Cl}(\mathrm{n}, 2), \mathrm{Gsp}(\mathrm{n}, 2))] \ldots \ldots \ldots \ldots \ldots . . .[(\mathrm{Cl}(\mathrm{n}, \mathrm{m} 1)$,

$\left.\left.\operatorname{Gsp}\left(n, m_{2}\right)\right)\right]$

\section{Consideration No. 3}

Let us consider a database DB_GEN (General Database) which consists of (n) database tables postulated as follows:

- DBGE (0) - database table which contains a set of principal data related to any object and is considered to be the database leading table. 


\section{Acta Technologica Dubnicae}

volume 1, 2011, issue 1

- DB_NO1 - database table which contains a set of data, the database table is subordinated to DBGE - database table.

- DB_NO2 - database table which contains a set of data, the database table is subordinated to DBGE - database table.

......................

- DB_N $\left(\mathrm{m}_{1}\right)$ - database table which contains a set of data, the database table is subordinated to DBGE - database table.

The database to be investigated contains one hierarchical level only (see also Figure 2). The DBGE-database is represented by an image which is denoted DBGEdatabase, while the image consists of (m1) segments postulated as follows:

- DBTGE (1) _Img - image segment which corresponds to DBGE-database table.

- DBTGE (2) _Img - image segment which corresponds to DBGE-database table.

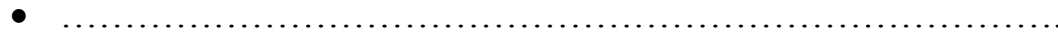

- DBTGE $\left(\mathrm{m}_{1}\right)_{\text {_Img }}$ - image segment which corresponds to DBGE-database table

and the formulas $(1.3 \mathrm{a} \ldots 1.3 \mathrm{e})$ may be postulated as a result of that

Image $=\left\{\operatorname{Seg}(1), \operatorname{Seg}(2) \ldots \operatorname{Seg}\left(m_{1}\right)\right\}$

Seg $(1)=$ DBGE $(0) \_$Img

Seg $(2)=$ DBGE (1)_Img

$\operatorname{Seg}(3)=\operatorname{DBGE}(2) \_\operatorname{Img}$

$\operatorname{Seg}(\mathrm{m} 1)=\operatorname{DBGE}\left(\mathrm{m}_{1}\right) \_$Img

Any of the above-mentioned segments represented by appropriate database tables consists of subordinated segments, while Seg(1) = DBTP_GE(0) is considered to be a leading segment or database table and the following three segments (see also formulas $1.3 \mathrm{c}, 1.3 \mathrm{~d}$ and $1.3 \mathrm{e}$ ) are considered to be the segments subordinated to segment Seg (1) - and the following formulas may be postulated:

$\operatorname{Seg}(1)=\left[\operatorname{Seg}(2) \ldots \operatorname{Seg}\left(m_{1}\right)\right]$

If formula (2.3b) is valid and Figure 2 is being respected, the following premise may be postulated: 
If $\operatorname{Seg}(1)=\operatorname{DBGE}(0) \_\operatorname{Img} \Rightarrow \operatorname{Seg}(1)=\Pi \operatorname{Seg}(i+1) \quad\left(\right.$ for $\left.\left.\mathrm{i}=1 \ldots . . m_{1}\right)\right) \& \operatorname{Seg}(i+1)=$ $=\Pi\{[\mathrm{Cl}(\mathrm{i}+1, \mathrm{j}), \mathrm{Gsp}(\mathrm{i}+1, \mathrm{j})]\}$ (for $\mathrm{i}=1 \ldots \mathrm{m}_{1}$ and $\left.\mathrm{j}=1 \ldots . . \mathrm{m}_{2}\right)$

while further appropriate assumptions shall be considered and respected

$\mathrm{Cl}(\mathrm{i}, 1)$ - contains DBTP - database table primary key

$\mathrm{Cl}(\mathrm{i}, \mathrm{j})$ - contains DBTP - database table foreign keys $(\mathrm{j}=1 \ldots \mathrm{k})$

$\mathrm{Cl}(\mathrm{i}, \mathrm{k}+1)$ - contains DBTP - database table attributes $\left(\mathrm{k}=1 \ldots . \mathrm{m}_{2}\right)$

Let us introduce the following equation:

$\operatorname{Gsp}(i, j)=\{[(P K(i),(F K(i, j))],[(P K(i+1), F K(i+1, j)]\}$

For $i=1 \ldots . ., j=2 \ldots m_{1}$,

This equation is considered to be a deterministic image gasp structure representation and may be postulated with respect to formulas (1.5a) and (1.5b) while the entire image structure is represented by formula (2.7).

$\{\operatorname{Img}\}=\Pi\{[(\operatorname{Seg}(1)), \operatorname{Gsp}(i, j), \operatorname{Seg}(i)\}$

For $\mathrm{i}=1 \ldots . \mathrm{n}, \mathrm{j}=2 \ldots \mathrm{m}_{1}$

Now, let us analyze a segment denoted as Seg (1). The segment contains two subordinated clusters $\mathrm{Cl}(1,1), \mathrm{Cl}(1,2)$ and $\mathrm{Cl}(1,3)$. The first cluster, denoted as $\mathrm{Cl}(1,1)$ contains only one element which is represented by the primary key which is closely related to Seg (1). However, the second cluster, denoted as $\mathrm{Cl}(1,2)$ contains a foreign key which corresponds to number of segments subordinated to Seg (1) as well. The second cluster, denoted as $\mathrm{Cl}(1,3)$ contains attributes proposed by the database designer (see also Figure 2). Let us try to express any database image $\{\operatorname{Img}\}$ (see also formula (2.7) via model based on linguistic approach which is based on existing of Tbe, Pet a Ret terms (see also section 2.1). The Pet terms are considered to be the Principal terms which the Tbe (terms to be explained) are derived or generated from. The above-mentioned Pet terms are closely related to $\mathrm{Cl}(1,1)$ and $\mathrm{Cl}(1,2)$ content, while the following formulas may be postulated:

Pet $(1,1)=[\operatorname{Pk}(1), F k(1,1)]$

$\mathrm{Cl}(1,1)=\operatorname{Pet}(1,2)=[\operatorname{Pk}(1), \operatorname{Fk}(1,2)]=\operatorname{Pet}(\mathrm{i}, \mathrm{j})=\{[\operatorname{Pk}(\mathrm{i}), \operatorname{Fk}(\mathrm{i}, \mathrm{j})$

For $\mathrm{i}=1$ and $\mathrm{j}=1 \ldots . \mathrm{n}$

Pet $(1, \mathrm{n})=[\mathrm{Pk}(1), \mathrm{Fk}(1, \mathrm{n})]$

$\mathrm{Cl}(1,2)=\operatorname{Pet}(2,1)=[\operatorname{Pk}(2), \operatorname{Fk}(2,1)]$

$\mathrm{Cl}(1,3)=\operatorname{Pet}(3,1)=[\operatorname{Pk}(3), \operatorname{Fk}(3,1)]$ 
$\mathrm{Cl}(1, \mathrm{n})=\operatorname{Pet}(\mathrm{n}, 1)=[\mathrm{Pk}(\mathrm{n}), \mathrm{Fk}(\mathrm{n}, 1)]$

Tbe $(\mathrm{i}, \mathrm{j})=\mathrm{Cl}(1,1) \otimes \mathrm{Cl}(1, \mathrm{n})=\{[\operatorname{Pk}(\mathrm{i}), \mathrm{Fk}(\mathrm{i}, \mathrm{j})]\} \otimes\left\{\left[\operatorname{Pk}\left(\mathrm{i}^{\prime}\right), \operatorname{Fk}\left(\mathrm{i}^{\prime}, \mathrm{j}^{\prime}\right)\right]\right\}$

Tbe $(1,2)=[\operatorname{Pk}(1), \operatorname{Fk}(1,1)] \otimes \operatorname{Pk}(2), \operatorname{Fk}(2,1)]=[(\operatorname{Pk}(1) \otimes \operatorname{Pk}(2))],[(\operatorname{Fk}(1,1)$

$\otimes \mathrm{Pk}(2))],[(\mathrm{Pk}(1) \otimes \mathrm{Fk}(2,1))],[(\mathrm{Fk}(1,1) \otimes \mathrm{Pk}(2))]$

$[(\mathrm{Pk}(1) \otimes \mathrm{Pk}(2))]=\varnothing-$ this product has no semantic meaning;

$[(\mathrm{Fk}(1,1) \otimes \mathrm{Pk}(2))]=\varnothing-$ this product has no semantic meaning;

$[(\mathrm{Pk}(1) \otimes \mathrm{Fk}(2,1))] \neq \varnothing-\operatorname{Pet}(1,2)$ meaningful semantic relation

between Seg (1) and Seg (2);

$[(\mathrm{Fk}(1,1) \otimes \mathrm{Pk}(2))]=\varnothing-$ this product has no semantic meaning;

Similar formulas may be derived, generated and interpreted for further relations which are described within the Section 2 of the paper.

\subsection{Terms and principles related to image and text semantic analysis systems applied in e-learning systems}

In general, Image and Text Semantic Analysis Systems cover a large area when considering their practical application while semantic analysis of images may be applied to analyze different types of images (deterministic or non-deterministic) as well. However, an image structure may be described via pre-defined deterministic relations and the type of images is denoted as deterministic images, the image structure which can be described via stochastic or heuristic relations is denoted as nondeterministic images. Both types of images may be applied in educational or teaching processes. The deterministic images may be applied in teaching courses closely related to management or business processes and their information support, like the business process modeling and their information support modeling, e.g., problems of database models especially, where e-learning system plays an important role, are discussed in this paper. The set of steps, the principles related to the Image and Text Semantic Analysis Systems, when providing database conceptual, logical and physical model and when explaining it within the appropriate course are also analyzed here. However, we are doing our best to answer the question: "Why the Image and Text Semantic Analysis Systems are considered to be an important part of E-learning Systems?" 


\section{Design and implementation of image and text semantic analysis applications and systems related to deterministic images}

\subsection{Example of design concerned to conceptual model related to any simple one-level database}

Let us consider a database DB_Pers (Database Person) which consists of four database tables postulated as follows:

- DBTP - database table which contains principal data related to any personality and is considered to be the database leading table.

- DBTE - database table which contains data concerned to education for any personality, the principal date of whom are being stored in the DBTP database table.

- DBTS - database table which contains data concerned to branch of specialization for any personality, the principal date of which are being stored in the DBTP database table.

- DBTL - database table which contains data concerned to foreign language knowledge for any personality, the principal data of which are being stored in the DBTP database table.

The DB_Pers database is represented by the image which is denoted as DB_Pers_Img, while the image consists of four segments postulated as follows:

- DBTP_Img - image segment which corresponds to DBTP-database table.

- DBTE_Img - image segment which corresponds to DBTE-database table.

- DBTS_Img-image segment which corresponds to DBTS-database table.

- DBTL_Img-image segment which corresponds to DBTL-database table.

The formulas $(2.1 \mathrm{a} \ldots 2.1 \mathrm{e})$ may be postulated as a result of that.

$$
\begin{aligned}
& \text { Image }=\{\operatorname{Seg}(1), \operatorname{Seg}(2), \operatorname{Seg}(3), \operatorname{Seg}(4)\} \\
& \text { Seg }(1)=\text { DBTP_Img } \\
& \text { Seg }(2)=\text { DBTE_Img } \\
& \text { Seg }(3)=\text { DBTS_Img } \\
& \text { Seg }(4)=\text { DBTL_Img }
\end{aligned}
$$




\section{Acta Technologica Dubnicae}

volume 1, 2011, issue 1

Any of the above-mentioned segments represented by the appropriate database tables consists of subordinated segments, while Seg (1) = DBTP_Img is considered to be a leading segment or database table and the following three segments (see also formulas $1.1 \mathrm{c}, 2.1 \mathrm{~d}$ and 2.1e) are considered to be the segments subordinated to segment Seg (1) - see also formula (1b) and the following formula may be postulated:

$\operatorname{Seg}(1)=[\operatorname{Seg}(2), \operatorname{Seg}(3), \operatorname{Seg}(4)]$

$\operatorname{Seg}(1)=[\operatorname{Rel}(1,2), \operatorname{Seg}(2)]$

Seg $(1)=[\operatorname{Rel}(1,3), \operatorname{Seg}(3)]$

$\operatorname{Seg}(1)=[\operatorname{Rel}(1,4), \operatorname{Seg}(4)]$

Any of the segments denoted as Seg (2), Seg (3) or Seg (4) consists of appropriate clusters (see also Figure 1) while the following formulas may be postulated:

$\operatorname{Seg}(1)=[C l(1,1), C l(1,2), C l(1,3)]$

where

$\mathrm{Cl}(1,1)$ - contains DBTP-database table primary key $\mathrm{Cl}(1,2)$ - contains DBTP-database table foreign keys $\mathrm{Cl}(1,3)$ - contains DBTP-database table attributes $\operatorname{Seg}(2)=[\mathrm{Cl}(2,1), \mathrm{Cl}(2,2), \mathrm{Cl}(2,3)]$

where

$\mathrm{Cl}(2,1)$ - contains DBTE-database table primary key $\mathrm{Cl}(2,2)$ - contains DBTE-database table foreign key $\mathrm{Cl}(2,3)$ - contains DBTE-database table attributes $\operatorname{Seg}(3)=[\mathrm{Cl}(2,1), \mathrm{Cl}(2,2), \mathrm{Cl}(2,3)]$

where

$\mathrm{Cl}(3,1)$ - contains DBTS-database table primary key $\mathrm{Cl}(3,2)$ - contains DBTS-database table foreign key $\mathrm{Cl}(3,3)$ - contains DBTS-database table attributes $\operatorname{Seg}(4)=[\mathrm{Cl}(4,1), \mathrm{Cl}(4,2), \mathrm{Cl}(4,3)]$

where

$\mathrm{Cl}(4,1)$ - contains DBTL-database table primary key 


\section{Acta Technologica Dubnicae}

volume 1, 2011, issue 1

$\mathrm{Cl}(4,2)$ - contains DBTL-database table foreign key

$\mathrm{Cl}(4,3)$ - contains DBTL-database table attributes

Let us introduce the following equation:

Tbe $(i, j)=\mathrm{Cl}(1,1) \otimes \mathrm{Cl}(1, \mathrm{n})=\{[\mathrm{Pk}(\mathrm{i}), \mathrm{Fk}(\mathrm{i}, \mathrm{j})]\} \otimes\left\{\left[\mathrm{Pk}\left(\mathrm{i}^{\prime}\right), \mathrm{Fk}\left(\mathrm{i}^{\prime}, \mathrm{j}^{\prime}\right)\right]\right\}$

We apply it for the above-mentioned database type in order to establish the adequate relations between the leading database table and the database table subordinated to it. There may be found two types of relations:

a) Relations which imply a proper functionality of the designed database and are denoted as meaningful relations or relations with adequate semantic meaning, while a product value by which the actual relation is represented is not an empty set $(\neg \varnothing)$.

b) Relations which imply non-proper functionality of the designed database and are denoted as meaningless relations or relations with non-adequate semantic meaning, while a product value by which the actual relation is represented is an empty set $(\varnothing)$.

The following set of equations may be postulated as the result of that:

Tbe $(1,2)=[\operatorname{Pk}(1), \operatorname{Fk}(1,1)] \otimes \operatorname{Pk}(2), \operatorname{Fk}(2,1)]=[(\operatorname{Pk}(1) \otimes \operatorname{Pk}(2))],[(\operatorname{Fk}(1,1)$

$\otimes \mathrm{Pk}(2))],[(\mathrm{Pk}(1) \otimes \mathrm{Fk}(2,1))],[(\mathrm{Fk}(1,1) \otimes \mathrm{Pk}(2))]$

$[(\mathrm{Pk}(1) \otimes \mathrm{Pk}(2))]=\varnothing-$ the product has no semantic meaning

$[(\mathrm{Fk}(1,1) \otimes \mathrm{Pk}(2))]=\varnothing-$ the product has no semantic meaning

$[(\mathrm{Pk}(1) \otimes \mathrm{Fk}(2,1))] \neq \varnothing-\operatorname{Pet}(1,2)$ meaningful semantic relation

between Seg (1) and Seg (2) (the same corrections for 3.5b-c)

$[(\mathrm{Fk}(1,1) \otimes \mathrm{Pk}(2))]=\varnothing-$ this product has no semantic meaning

$\operatorname{Tbe}(1,2)=[(P k(1) \otimes F k(2,1))] \neq \varnothing-\operatorname{Gsp}(1,2)$

Tbe $(1,3)=[\mathrm{Pk}(1), \mathrm{Fk}(1,3)] \otimes \mathrm{Pk}(3), \mathrm{Fk}(3,1)]=[(\mathrm{Pk}(1) \otimes \mathrm{Pk}(3))]$,

$[(\mathrm{Fk}(1,1) \otimes \mathrm{Pk}(3))],[(\mathrm{Pk}(1) \otimes \mathrm{Fk}(3,1))],[(\mathrm{Fk}(1,1) \otimes \mathrm{Pk}(3))]$

$[(\mathrm{Pk}(1) \otimes \mathrm{Pk}(3))]=\varnothing-$ this product has no semantic meaning

$[(\mathrm{Fk}(1,1) \otimes \mathrm{Pk}(3))]=\varnothing-$ this product has no semantic meaning

$[(\mathrm{Pk}(1) \otimes \mathrm{Fk}(3,1))] \neq \varnothing-\mathrm{Pet}(1,3)$ meaningful semantic relation

between Seg (1) and Seg (2)

$[(\mathrm{Fk}(1,1) \otimes \mathrm{Pk}(3))]=\varnothing$ - this product has no semantic meaning

$\operatorname{Tbe}(1,3)=[(P k(1) \otimes F k(3,1))] \neq \varnothing-\operatorname{Gsp}(1,3)$ 
Tbe $(1,4)=[\mathrm{Pk}(1), \mathrm{Fk}(1,4)] \otimes \mathrm{Pk}(4), \mathrm{Fk}(4,1)]=[(\mathrm{Pk}(1) \otimes \mathrm{Pk}(4))]$, $[(\mathrm{Fk}(1,1 \otimes \mathrm{Pk}(4))],[(\mathrm{Pk}(1) \otimes \mathrm{Fk}(4,1))],[(\mathrm{Fk}(1,1) \otimes \mathrm{Pk}(4))]$

$[(\mathrm{Pk}(1) \otimes \mathrm{Pk}(4))]=\varnothing-$ this product has no semantic meaning

$[(\mathrm{Fk}(1,1) \otimes \mathrm{Pk}(4))]=\varnothing-$ this product has no semantic meaning

$[(\mathrm{Pk}(1) \otimes \mathrm{Fk}(4,1))] \neq \varnothing$ - Pet $(1,3)$ meaningful semantic relation

between Seg (1) and Seg (2)

$[(\mathrm{Fk}(1,1) \otimes \mathrm{Pk}(4))]=\varnothing$ - this product has no semantic meaning

$\operatorname{Tbe}(1,4)=[(P k(1) \otimes F k(4,1))] \neq \varnothing-G s p(1,4)$

When looking at formulas and equations denoted as $(2.5 \mathrm{a}-2.5 \mathrm{c})$, we can see, that meaningful relations may be represented by Tbe terms based on the primary key related to leading database table and foreign key related to the database table which is strictly subordinated to the leading database table (see also formulas $(2.5 \mathrm{a}-2.5 \mathrm{c})$. In general, any Tbe-term consists of more Pet and Ret terms, while the following equation may be postulated:

Tbe $(i, j)=f[P e t(i, j), \operatorname{Ret}(i, j)]$

With respect to the previous formulas $(2.5 \mathrm{a}-2.5 \mathrm{c})$, formula (2.6) may be postulated as follows:

Tbe $(1,4)=\operatorname{Pet}(1,4) \otimes \operatorname{Ret}(1,4)$

When considering a database image which consists of segments and gasps (see also formula (2.7) and the database image is considered to be a deterministic image, any Tbe terms are closely related to the image gasp, denoted as Gsp (i, j), while formula (2.8) may be postulated:

Tbe $(1,4)=\operatorname{Gsp}(1,4)$

Because of that, any Tbe term consists of Pet and Ret term and formula (2.7) is valid, the following formulas may be postulated:

Tbe $(1,4)=\operatorname{Pet}(1,4) \otimes \operatorname{Ret}(1,4)$

Pet $(1,4) \otimes \operatorname{Ret}(1,4)=\operatorname{Rel}(1,4)$

Tbe $(1,4)=\operatorname{Rel}(1,4)=\operatorname{Gsp}(1,4)$

With respect to the previous considerations, formulas and equations the following assertions or statements may be postulated: 
Any deterministic image may be represented by segments with an appropriate predefined structure based on adequate clusters and gasps, while any cluster consists of one unique (primary) attribute, more non-unique (foreign) attributes and further attributes which represent the cluster content as well. On the other hand, any gasp which creates an integral part of any deterministic image contains relations based on one unique (primary) attribute and one non-unique (foreign) attribute and provides an interconnection between the leading image segment and subordinated image segments. However, with respect of previous formulas and equations which indicate that, the above mentioned structure may be represented by Tbe, Pet and Ret terms, which create the basis for linguistic approach to deterministic image representation.

\section{Assertion No.2}

The results concentrated in Assertion No.1 entitle us to postulate the fact, that the previous formulas and equations may be applied in designing a database conceptual model, data warehouse conceptual model or when designing models of business processes.

\subsection{How image and text semantic analysis principles can be applied in teaching database course}

Previous section has dealt with the quantification of deterministic images. The simple database conceptual model can be considered to be such an image. In Section 2.3, there is postulated the following question: "Why Image and Text Semantic Analysis Systems are considered to be important part of E-learning Systems?" Let us try to find an answer to this question.

We should think in two directions when looking for the answer. The first direction is closely related to database conceptual model design and the second direction is concerned to check and control of the database conceptual model design, especially when looking for an answer to the following question. "Are primary and foreign keys, which create an integral part of any database table design together with appropriate relations proposed correctly and is their functionality suitable?"

When looking for an answer to the first direction, the database designer is required to determine a number of database tables which should create the total database content image and establish the leading database table. After that, he/she is able to get the answer how the leading database table shall be designed from primary key view and 


\section{Acta Technologica Dubnicae}

volume 1, 2011, issue 1

how many foreign keys shall be contained in the database table. On the other hand, when considering the database tables subordinated to the leading database table, the designer gets the appropriate information how the primary and foreign keys shall look like in order to create an adequate relation properly.

Finally, the designer gets the information how the actual relation shall be designed correctly. When looking for an answer to the second direction, the database designer is required to present the actual database conceptual model together with appropriate primary and foreign keys and also with adequate relations among actual database tables in order to evaluate semantic meaning and correctness of a designed relation which plays a role of principal importance within any database conceptual model, while the formulas $(2.5 \mathrm{a}-2.5 \mathrm{c})$ are important for these purposes. On the other hand, the design and implementation of an appropriate application program operating based on this algorithm is a matter of principal importance.

\section{Conclusion}

The paper presented deals with the application of image semantic analysis principles in educational and teaching process, especially how these principles may be applied when explaining problems of simple database conceptual model. With respect to the requirement, a special type of image denoted as a deterministic image was postulated. Its clusters and gasps have an appropriate pre-defined structure described via adequate deterministic relations.

As a result of that, an adequate algorithm related to the design and implementation of simple database conceptual model when considering construction of appropriate relations based on primary and foreign keys, where the students - beginners in these branches, make most mistakes. However, the information in Assertion No.1 and Assertion No. 2 together with information in Section 3.2 can be also useful for them. The designed algorithm can be further developed and applied for more complicated database conceptual models.

\section{References}

BOLSHAKOV, I., GELBUKH, A. Computer Linguistics - Models, Resources, Applications. http://www.gelbukh.com/clbook/Computational-Linguistics.htm

DOWNIE, J. S. Text Analysis. http://lucian.uchicago.edu/blogs/dhcs2008/category/text-analysis/

EAKINS, J., GRAHAM, M. Content-based Image Retrieval. http://www.buddies.org/articles/THESIS.DOC 


\section{Acta Technologica Dubnicae}

volume 1, 2011, issue 1

HARRIS, R.: Semantics 1. http://www.virtualsalt.com/think/semant1.htm

KOŠIR, A., TAŠIC, J. Formal system for digital image scene analysis. http://Idos.fe.unilj.si/docs/documents/20021009132908_andrejk.pdf

NIEMEYER, I., CANTY, M. J. Knowledge-Based Analysis of Change Images by Object Oriented Post Classification. http://ww.innemeyer.de/publications/urs01nie.pdf

STAŠÁK, J. 2004a. Application of Fuzzy Sets Apparatus in Image Semantic Analysis. In Proceedings of the 5th International Carpathian Control Conference. Krakow: Faculty of Mechanical Engineering and Robotics, 2004, p. 255-260.

STAŠÁK, J. 2004b. A Contribution to Semantic Text Analysis (Section 2.1 and 2.2). In Electronic Computers and Informatics ECI. Košice: The University of Technology, Department of Computers and Informatics of FEI, 2004, p.132-144.

STAŠÁK, J. 2004c. Data and Information Delivery Evaluation and Modeling Using Fuzzy Sets. In Acta Facultatis Aerarii Publici, vol.1-2, 2004, p. 268-274.

STAŠÁK, J. 2004d. A Contribution to Image Semantic Analysis. In Informace na dlani. Praha: Albertina Income Praha s.r.o., 2004.

STAŠÁK, J. 2005. Image Databases Designed and Implemented Based on Fuzzy Set Algorithms. In Proceedings of 6th International Carpathian Control Conference ICCC 205, volume I. Miskolc-Lillafured, 2005, p. 419-430.

STAŠÁK, J. 2006. Modeling of Text Semantic with the use of Fuzzy Sets. In Ekonomičny vistnik NTUU, 2006, n. 3, p.376-384.

The Visual Literacy White Paper.

http://www.adobe.com/uk/education/pdf/adobe_visual_literacy_paper.pdf 